\title{
Variation saisonnière de l'activité phosphatase alcaline associée au phytoplancton de la retenue hypereutrophe de Grangent (Loire)
}

\author{
H. Giraudet ${ }^{1}$ \\ D. Abrial ${ }^{1}$ \\ J.-L. Berthon ${ }^{1}$ \\ B. Buisson ${ }^{1}$
}

Mots-clés : retenue hypereutrophe, phytoplancton, Cyanobactérie, activité phosphatase alcaline, phosphore.

L'évolution spatio-temporelle de l'activité phosphatase alcaline spécifique (APA) (EC: 3.1.3.1) des peuplements phytoplanctoniques de la retenue de Grangent (Loire) a été étudiée. Cette activité enzymatique est maximale en été (comprise entre 20 et $69,4 \mu \mathrm{mol} \mathrm{P}-\mathrm{PO}_{4}{ }^{3-} \cdot \mathrm{h}^{-1} \cdot \mathrm{mg}^{-1}$ au niveau de l'épilimnion) et minimale en automne et au printemps (variant de 0,1 à $10 \mu \mathrm{mol} \mathrm{P}$ $\mathrm{PO}_{4}{ }^{3-} \cdot \mathrm{h}^{-1} \cdot \mathrm{mg}^{-1}$ ). Elle diminue au fur et à mesure que l'on s'enfonce dans la colonne d'eau et est inversement corrélée à la teneur en orthophosphate du milieu. Par ailleurs, l'activité phosphatasique est étroitement liée aux fleurs d'eau cyanobactériennes. En présence d'une même concentration en orthophosphate, les algues semblent être de meilleures compétitrices que les Cyanobactéries dans l'assimilation du $\mathrm{PO}_{4}{ }^{3-}$ dissous. Les Cyanobactéries ont ainsi davantage recours à l'activité phosphatasique pour disposer du phosphore minéral indispensable à leur métabolisme. Cette différence de comportement dans l'utilisation du phosphore entre les Cyanobactéries et les algues pourrait représenter un critère explicatif supplémentaire des successions écologiques.

\section{Seasonal variation of the alkaline phosphatase activity of phytoplankton in the hypereutrophic Grangent reservoir (Loire)}

Keywords : hypereutrophic reservoir, phytoplankton, Cyanobacterium, alkaline phosphatase activity, phosphorus.

The seasonal and spatial changes of the specific alkaline phosphatase activity (APA) (EC: 3.1.3.1) were studied in relation to several abiotic and biotic factors in the Grangent reservoir (located on the Loire River). The enzymatic activity was maximal in summer (between 20 and $69,4 \mu \mathrm{mol} \mathrm{P}-\mathrm{PO}_{4}{ }^{3-} \cdot \mathrm{h}^{-1} \cdot \mathrm{mg}^{-1}$ in the epilimnion) and minimal in spring and in autumn (varying from 0,1 to $10 \mu \mathrm{mol} \mathrm{P}-\mathrm{PO}_{4}{ }^{3-} \cdot \mathrm{h}^{-1} \cdot \mathrm{mg}^{-1}$ ). This activity was greatest in the epilimnion and became weaker with depth. APA appeared to be inversely related to the concentration of orthophosphate. Finally, the main peak of the alkaline phosphatase activity seemed to be strictly associated with blooms of Cyanobacteria. In the presence of a similar concentration of orthophosphate, algae showed a higher uptake rate of this nutrient than Cyanobacteria. Therefore, Cyanobacteria were more often obliged to resort to the phosphatase activity for mineralizing organic phosphorus. The observed differences in the phosphorus assimilation between algae and Cyanobacteria might explain ecological successions related to the seasonal variations.

\section{Introduction}

L'eutrophisation est devenue, ces dernières décennies, le problème des plans d'eau, des fleuves lents et plus particulièrement des réservoirs où elle s'accompagne fréquemment du développement de fleurs d'eau

\footnotetext{
1.Université J. Monnet, Laboratoire de Biologie Animale et Appliquée, 23 rue du Dr. P. Michelon, 42023 Saint-Etienne Cedex, France.
}

cyanobactériennes (Heath \& Cooke 1975, Wynne 1981, Siuda \& Chrost 1987, Devaux 1993, Michard 1994, Jamet et al. 1995, Berthon et al. 1996). L'implication du phosphore dans la prólifération phytoplanctonique et dans l'apparition et le dévèloppement des fleurs d'eau cyanobactériennes a été mise en évidence (Devaux 1993, Michard 1994, Jamet et al. 1995, Berthon et al. 1996). L'ion orthophosphate est la seule forme de phosphore directement assimilable par les organismes. Cependant, une carence, voire même une ab- 
sence de $\mathrm{PO}_{4}{ }^{3-}$ dissous, n'est pas obligatoirement le facteur limitant d'une communauté phytoplanctonique. En effet, la plupart des espèces disposent d'enzymes, les phosphatases, capables de transformer le phosphore organique en phosphore minéral. Les phosphatases, également appelées phosphomonoestérases ou phosphomonoesterhydrolases (E.C : 3.1.3.1), hydrolysent les esters ou les anhydrides de l'acide phosphorique, libérant ainsi de l'orthophosphate (Feder 1973). De nombreux travaux ont montré que l'activité phosphatasique est indicatrice d'une carence en phosphore minéral (Boavida \& Heath 1986, Wynne \& Rhee 1986, Chrost \& Overbeck 1987, Siuda \& Chrost 1987, Lapointe \& O'Connel 1989, Feuillade et al. 1990, Hernandez et al. 1992, Perez \& Romero 1993). La synthèse de la phosphatase alcaline n'est déclenchée qu'à partir d'un seuil d'épuisement du stock phosphoré constitué par le phytoplancton. Elle dépend des espèces constitutives de la biocénose (Huber \& Hamel 1985, Hino 1988, Olsson 1991).

Dans le cadre d'une étude sur l'eutrophisation de la retenue de Grangent, nous avons étudié l'évolution spatio-temporelle de l'activité phosphatase alcaline des peuplements phytoplanctoniques au point SaintVictor-sur-Loire. Le but est de montrer si l'activité phosphatasique est indicatrice ou non d'une carence en phosphore minéral mais également si certaines espèces phytoplanctoniques possèdent une plus grande capacité d'utilisation des substrats organophosphorés comme source de phosphore minéral.

\section{Matériel et méthodes}

\subsection{Présentation du biotope}

La retenue de Grangent, mise en eau en 1957, est située dans le Sud-Est du Massif Central sur le cours amont de la Loire et à une quinzaine de kilomètres à l'Ouest de Saint-Etienne. Elle possède une capacité totale de $57,410^{6} \mathrm{~m}^{3}$ d'eau, s'engouffrant dans les gorges de la Loire sur une longueur de $21 \mathrm{~km}$. Sa largeur maximale atteint $400 \mathrm{~m}$ pour une superficie de 365 hectares et une profondeur moyenne de l'ordre de $35 \mathrm{~m}$. Les fonctions principales de cet ouvrage sont la production d'électricité, l'alimentation en eau potable de collectivités voisines de Saint-Etienne mais ce plan d'eau est également un site touristique (base nautique de Saint-Victor-sur-Loire, plages aménagées, pêche). Dès la fin des années 70 , le caractère eutrophe de la retenue, accompagné de la formation de fleurs d'eau à Microcystis aeruginosa, a été mis en évidence (Devaux 1993, Michard 1994, Jamet et al. 1995, Berthon et al. 1996). La présence massive de ces Cyanobacté- ries, en période estivale, montre un dysfonctionnement de la biocénose qui a abouti aujourd'hui à une hypereutrophisation de la retenue.

\subsection{Echantillonnage}

Les prélèvements ont été effectués d'avril 1996 (semaine 14) à fin octobre 1996 (semaine 44) suivant un pas hebdomadaire d'échantillonnage. Ils ont eu lieu les vendredis à heure fixe (11 heures), en un même point «Saint-Victor-sur-Loire» aux profondeurs - $0.5 \mathrm{~m},-$ $2.5 \mathrm{~m},-5 \mathrm{~m}$ et $-10 \mathrm{~m}$. Les échantillons ont été récoltés à l'aide d'une bouteille opaque de type Van Dorn d'un volume de 8 litres. L'eau prélevée a ensuite été préfiltrée sur filtre de $80 \mu \mathrm{m}$ de vide de maille afin d'éliminer la plus grande partie du zooplancton.

\subsection{Paramètres abiotiques et biotiques}

Le dosage des ions orthophosphates repose sur la formation en milieu acide d'un complexe coloré en bleu en présence de molybdate d'ammonium et de tartrate double d'antimoine et de potassium. La mesure spectrophotométrique a été effectuée à $700 \mathrm{~nm}$ et à $880 \mathrm{~nm}$ de longueur d'onde après filtration des échantillons sur membrane de porosité de $0,22 \mu \mathrm{m}$ (Norme AFNOR NF T90.023 ).

La biomasse phytoplanctonique a été déterminée à partir du dénombrement cellulaire effectué à l'aide d'un microscope inversé (Wild 40) suivant la méthode d'Utermöhl (1958) modifiée par Legendre \& Watt (1972). Les biomasses spécifiques ont été calculées en fonction des biovolumes cellulaires suivant la méthode de Lohman (1908) et sont exprimées en milligramme de Chlorophylle a/litre.

La teneur en Chlorophylle $a$ a été déterminée suivant la méthode spectrophotométrique de Lorenzen (1967) après filtration de 0,51 d'eau sur membrane Whatman $\mathrm{GF} / \mathrm{C}$ de porosité $1,2 \mu \mathrm{m}$.

Afin de déterminer l'activité enzymatique spécifique des peuplements phytoplanctoniques, la concentration en protéines de la fraction nano et microplantonique a été mesurée suivant la méthode de Lowry et al. (1951) après filtration de volumes de $250 \mathrm{ml}$ d'eau sur des membranes en fibre de verre Whatman GF/C.

\subsection{Activité phosphatase alcaline (APA)}

L'activité phosphatasique spécifique des cellules micro et nanoplanctoniques a été mesurée par la technique colorimétrique de Reichardt et al. (1967) utilisant $0,5 \mathrm{ml}$ de para-nitrophényl phosphate (pNPP) à 1 $\mathrm{mg} \cdot \mathrm{ml}^{-1}$ comme substrat chromogène. Des échantillons de $250 \mathrm{ml}$ d'eau homogénéisée filtrée sur mem- 
brane en fibre de verre Whatman GF/C ont été incubés 6 heures à $37^{\circ} \mathrm{C}$ en présence de pNPP. Ce substrat permet de quantifier la phosphatase alcaline par mesure de l'absorbance à une longueur d'onde de $410 \mathrm{~nm}$. Cette activité phosphatasique spécifique correspond à l'activité enzymatique exprimée en micromoles d'orthophosphate libérées par heure et par milligramme de protéines. Pour chaque échantillon, des triplicats de la mesure de l'absorbance ont été réalisés.

\subsection{Analyse statistique}

Afin de synthétiser l'ensemble des résultats, une Analyse en Composantes Principales, méthode mathématique décrite initialement par Hotelling (1933), a été réalisée. Cette méthode permet d'identifier une structure dans l'ensemble des résultats par partition de la variance en facteurs essentiels correspondant aux vecteurs propres extraits de matrices de corrélations. A partir de ces facteurs essentiels expliquant le maximum de variance, on recherche une corrélation avec des paramètres écologiques (Legendre \& Legendre 1979).

\section{Résultats}

\subsection{Paramètres abiotiques et biotiques}

Les concentrations en $\mathrm{PO}_{4}{ }^{3-}$ varient de 0,01 à 0,3 mg. $1^{-1}$ avec des maxima en automne sans doute causés par une augmentation des apports de la Loire, par une réduction de l'activité photosynthétique et surtout par le brassage automnal. Les valeurs minimales, quant à elles, sont estivales et trouvent leur origine dans la diminution des apports du fleuve en raison de l'étiage et dans l'utilisation de cet anion par le phytoplancton (Fig. 1).

Au printemps, la biomasse totale est largement dominée par la biomasse des Diatomées qui dépasse 25 mg.1 -1 $^{-1}$ (Fig. 2). Il s'agit alors principalement d'Asterionella formosa (de 8,5 $10^{6}$ à $1810^{6}$ cell.1-1 $^{-1}$ ) (Fig. 3).

Durant tout l'été, les Chlorophycées ou algues vertes et les Euglénophycées constituent en moyenne plus de 90\% de la biomasse totale. Les genres d'algues vertes les plus rencontrés sont Staurastrum, Scenedesmus et Chlamydomonas. Une poussée considérable mais fugace des Euglénophycées (Euglena viridis et Euglena polymorpha) le 22.08 .96 à $-5 \mathrm{~m}$ est à l'origine d'une
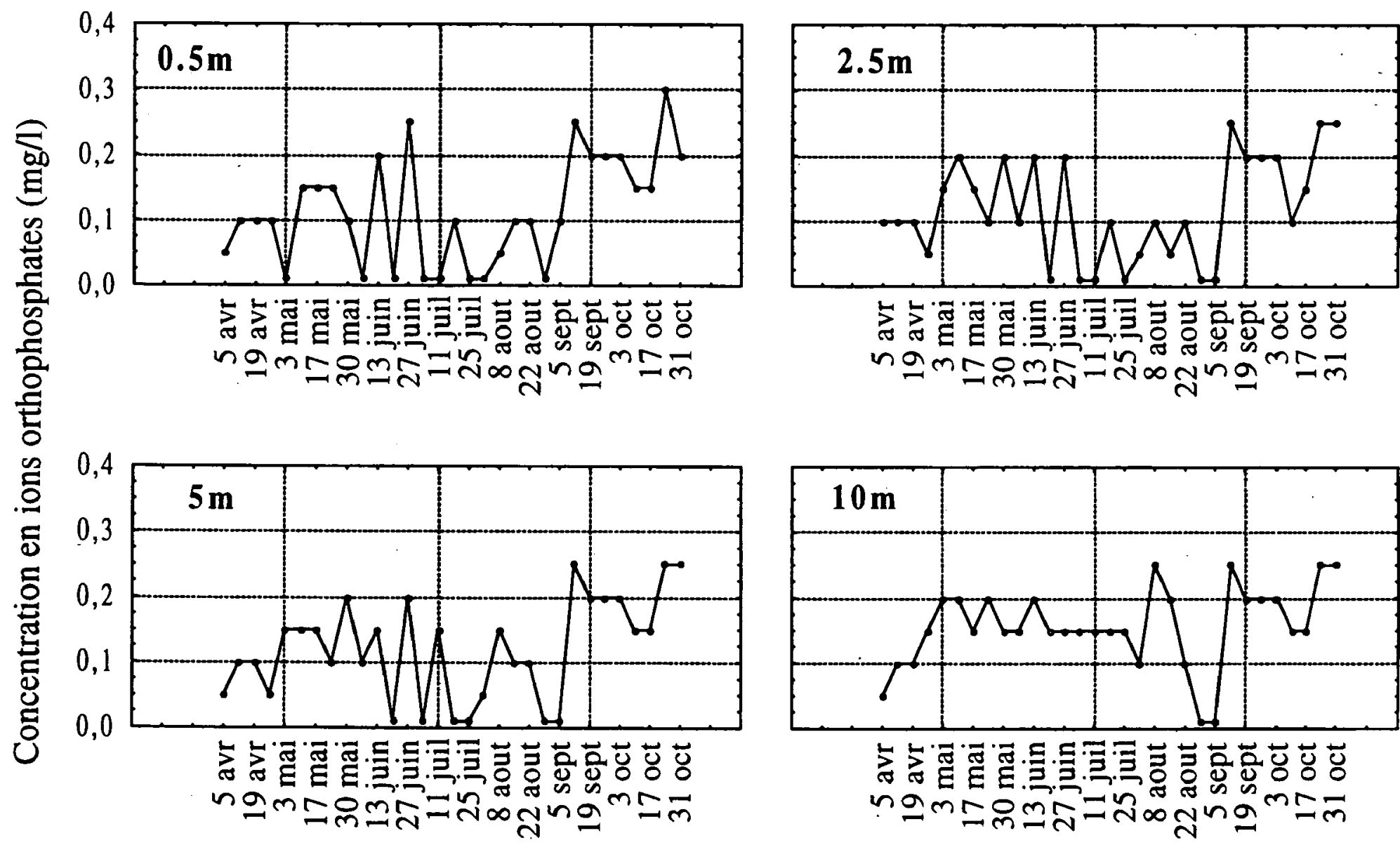

Fig. 1. Evolution spatio-temporelle de la concentration en ions orthophosphates.

Fig. 1. Temporal and spatial changes in the concentration of orthophosphate. 
biomasse exceptionnelle franchissant $200 \mathrm{mg} . \mathrm{l}^{-1}$ (Fig. 2).

En automne, les peuplements de Cyanobactéries et d'algues vertes périclitent laissant la place aux Diatomées déjà observées au printemps avec des biomasses comprises entre 0,32 et $16,7 \mathrm{mg} . \mathrm{l}^{-1}$ (Figs. 2, 3).

Le diamètre réduit des cellules cyanobactériennes explique leurs très faibles biomasses obtenues à partir des biovolumes. Durant cette étude, la biomasse des Cyanobactéries n'a jamais atteint $1 \mathrm{mg} . \mathrm{l}^{-1}$ c'est pourquoi elle est difficilement distinguable sur les figures.

Les teneurs en Chlorophylle $a$ varient de façon importante durant notre campagne puisque les valeurs extrêmes mesurées sont de 1,49 et $88,7 \mathrm{mg} \cdot \mathrm{m}^{-3}$. C'est début juillet et pendant les 3 premières semaines d'août, c'est-à-dire au moment des poussées de Cyanobactéries et d'algues vertes que l'on observe les valeurs les plus élevées. Les concentrations maximales sont comprises, le 04.07.96, entre $22,8 \mathrm{mg} \cdot \mathrm{m}^{-3}$ à $-5 \mathrm{~m}$ et 88,7 mg.m ${ }^{-3}$ à $-2,5$ m (Fig. 4).
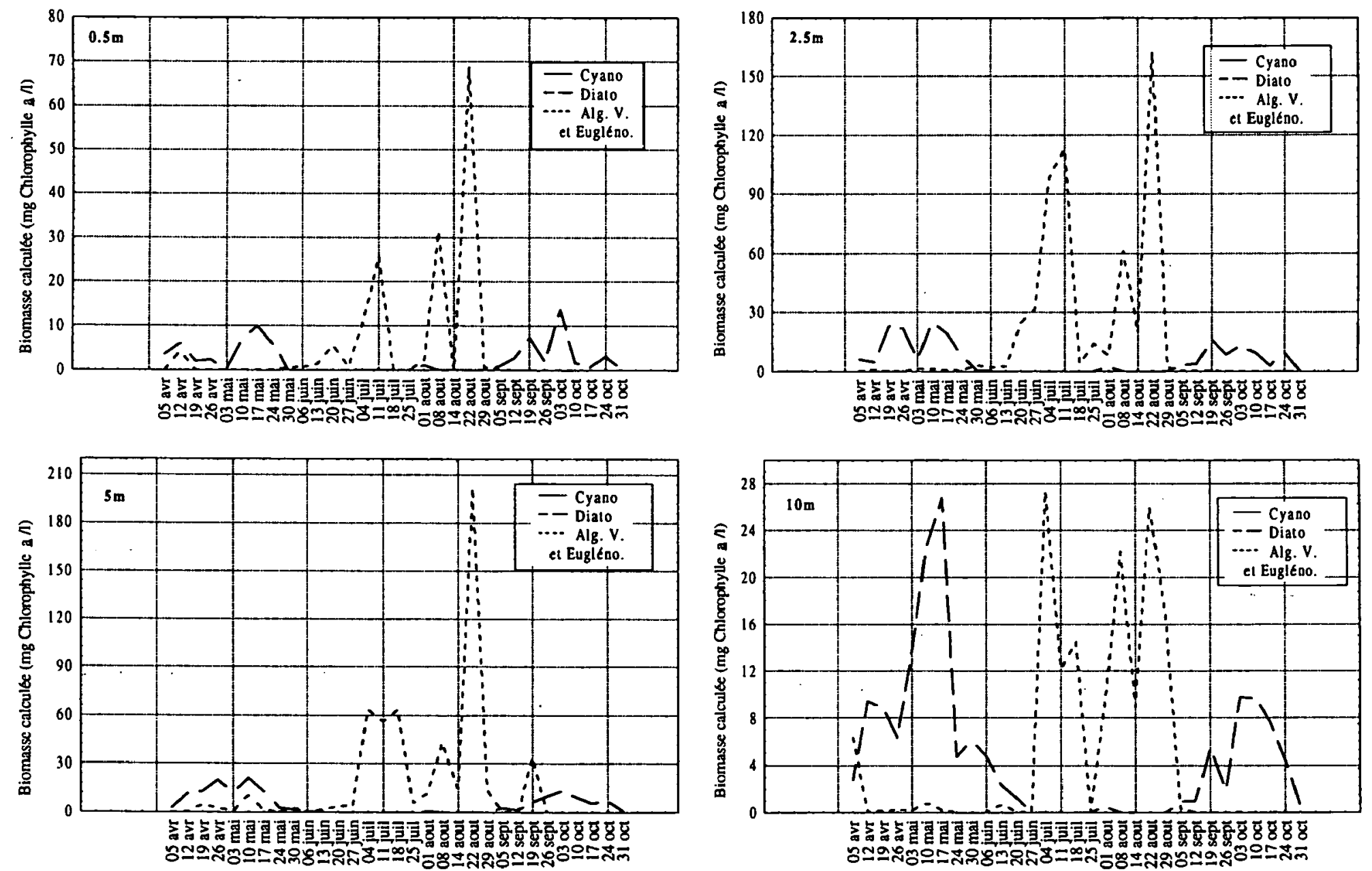

Les concentrations en protéines varient de 0,96 à $1,92 \mathrm{mg} . \mathrm{l}^{-1}$. Les plus grandes fluctuations sont observées dans les couches superficielles et ont lieu de mimai à fin août. C'est pendant cette période que les valeurs maximales sont mesurées $\left(1,92 \mathrm{mg} \cdot \mathrm{l}^{-1}\right.$ le 24.05.96 à $-0,5 \mathrm{~m}$ ). Les valeurs minimales sont plutôt obtenues au début de la campagne $\left(0,96 \mathrm{mg} .1^{-1}\right.$ le 05.04 .96 à $-0,5 \mathrm{~m}$ ) mais aussi dès l'arrivée de l'automne et du brassage (Fig. 5).

\subsection{Activité phosphatase alcaline (APA) :}

D'une façon générale, l'activité phosphatasique spécifique varie de 0,1 (le 30.05 .96 à - 2,5m) à $69,4 \mu \mathrm{mol}$

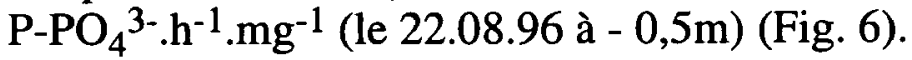

Une variation spatiale et temporelle de l'APA est observée :

- D'une part, l'activité phosphatasique spécifique est faible et relativement constante durant le printemps (toujours inférieure à $20 \mu \mathrm{mol} \mathrm{P}-\mathrm{PO}_{4}{ }^{3-} \cdot \mathrm{h}^{-1} \cdot \mathrm{mg}^{-1}$ ) et l'automne (ne dépassant pas $10 \mu \mathrm{mol} \mathrm{P}^{-\mathrm{PO}_{4}{ }^{3-} \text {. }}$ $\mathrm{h}^{-1} \cdot \mathrm{mg}^{-1}$ ) alors qu'elle fluctue davantage et atteint des

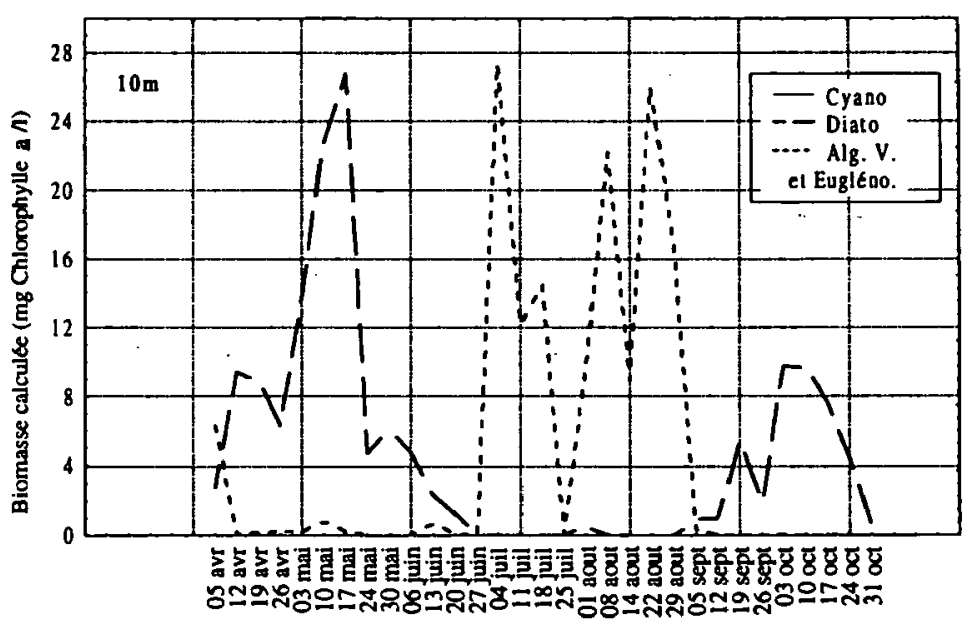

Fig. 2. Répartition spatio-temporelle de la biomasse phytoplanctonique. L'échelle des biomasses calculées change en fonction de la profondeur. Fig. 2. Spatial-temporal distribution of the phytoplanktonic biomass. Scale of the phytoplanktonic biomass change with depth. 

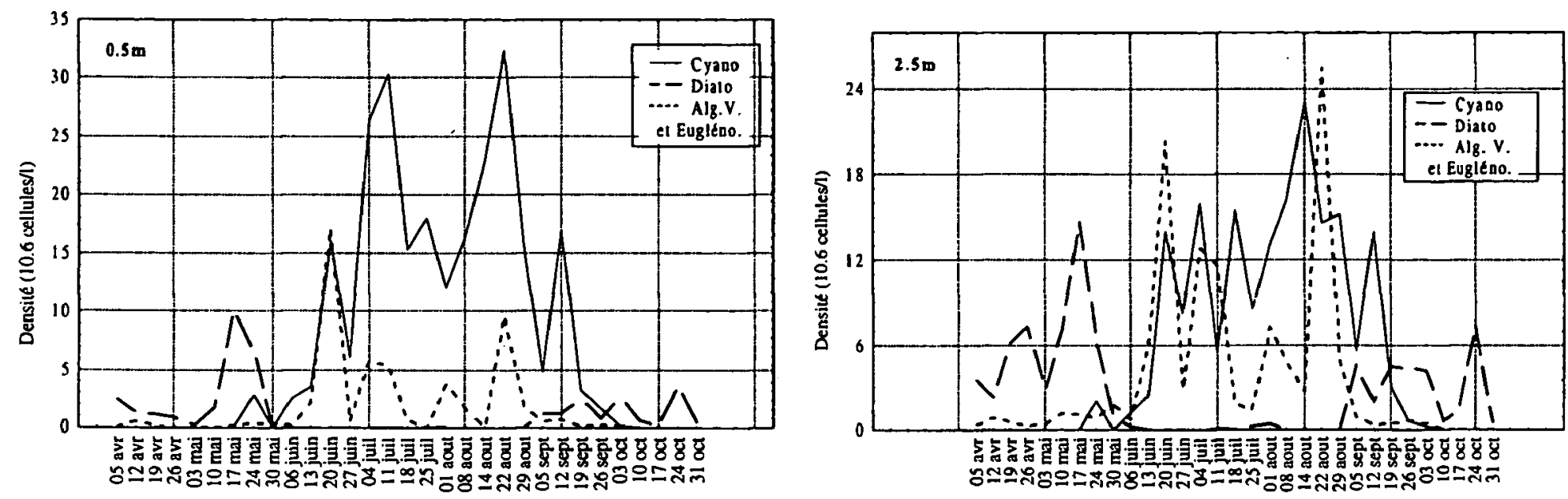

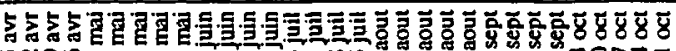

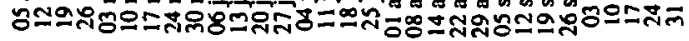
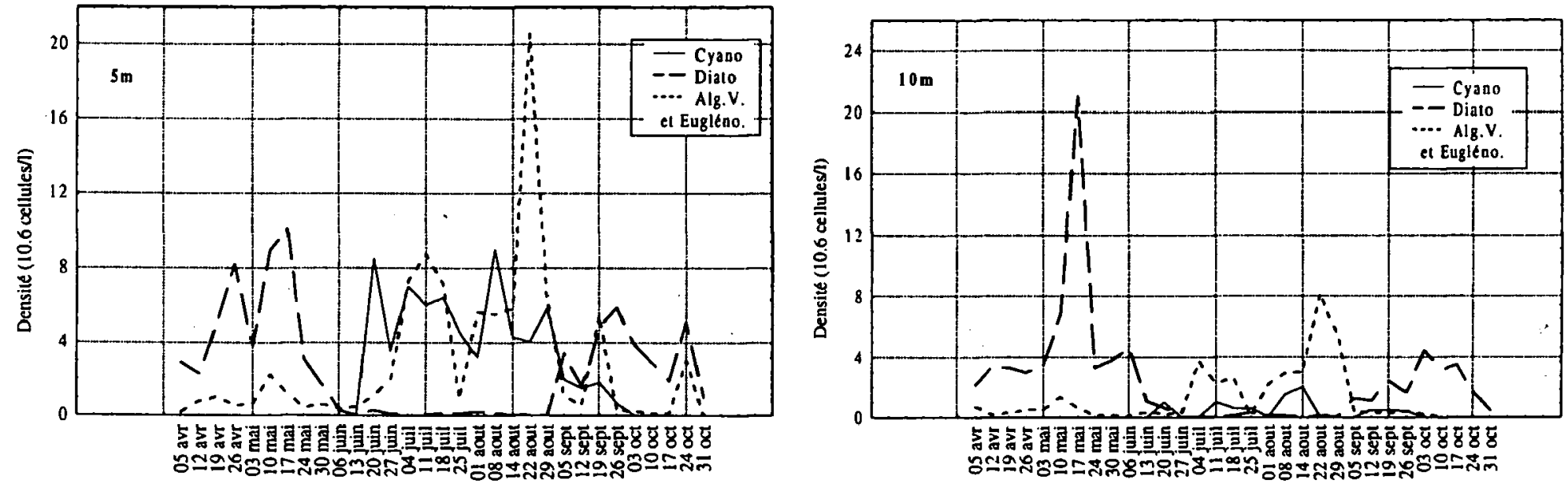

Fig. 3. Evolution spatio-temporelle de la densité phytoplanctonique. L'échelle des densités cellulaires change en fonction de la profondeur. Fig. 3. Spatial-temporal distribution of the phytoplanktonic cellular density. Scale of the phytoplanktonic cellular density change with depth.

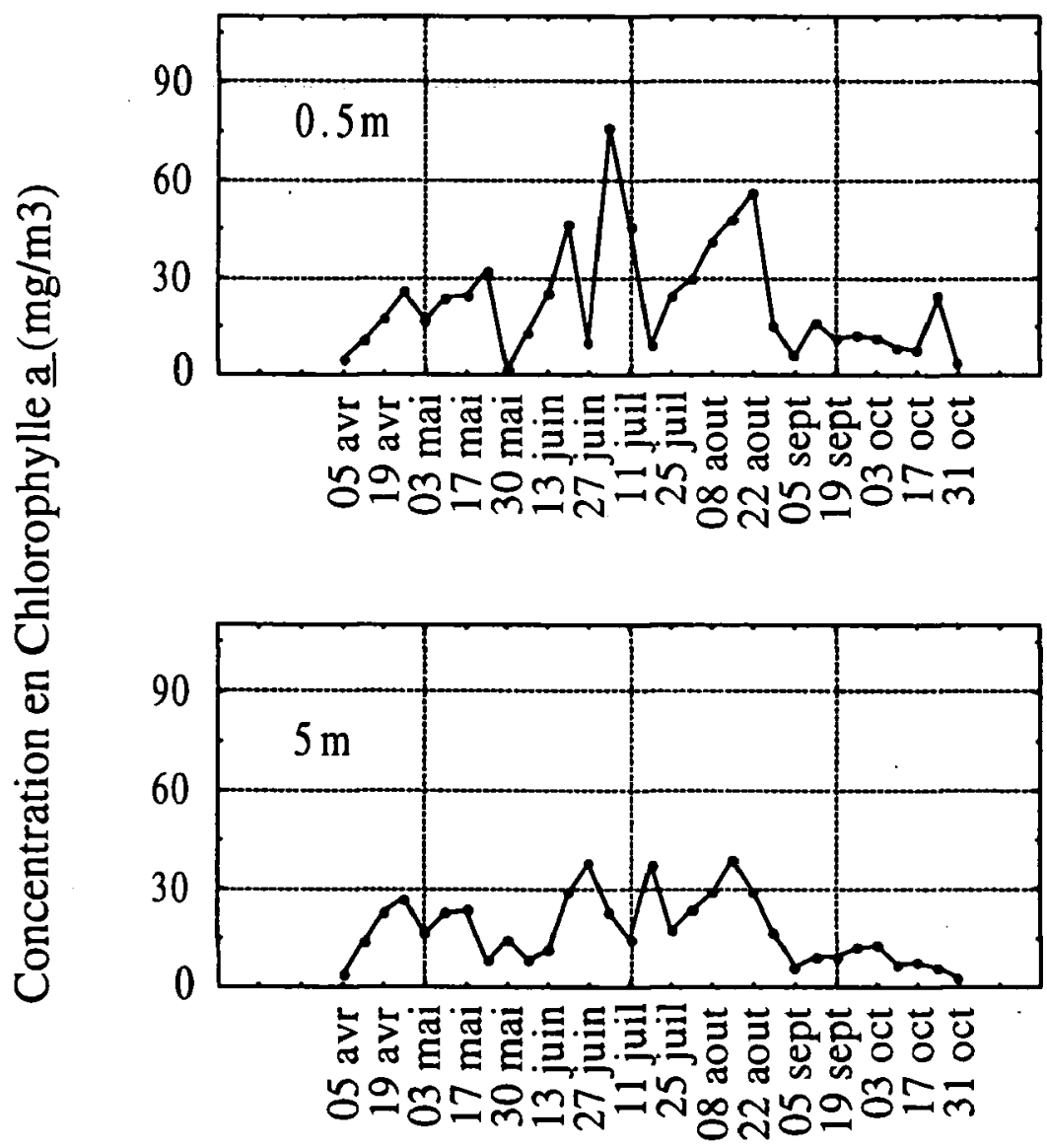

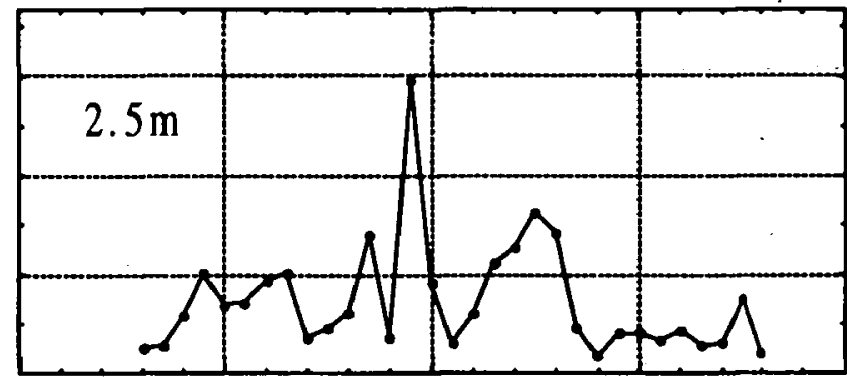

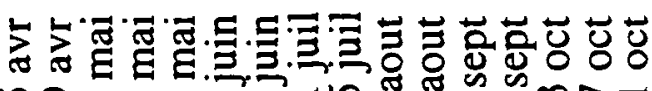

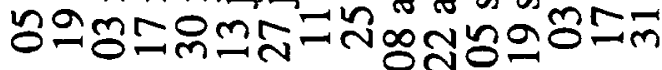

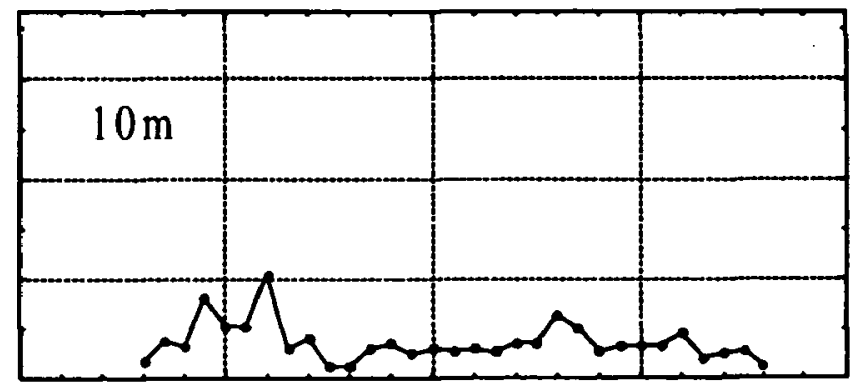

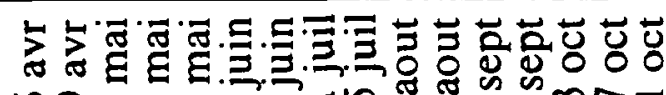

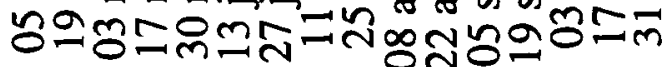

Fig. 4. Distribution spatio-temporelle de la teneur en Chlorophylle $a$.

Fig. 4. Spatial-temporal distribution of the Chlorophyll $a$ concentration. 

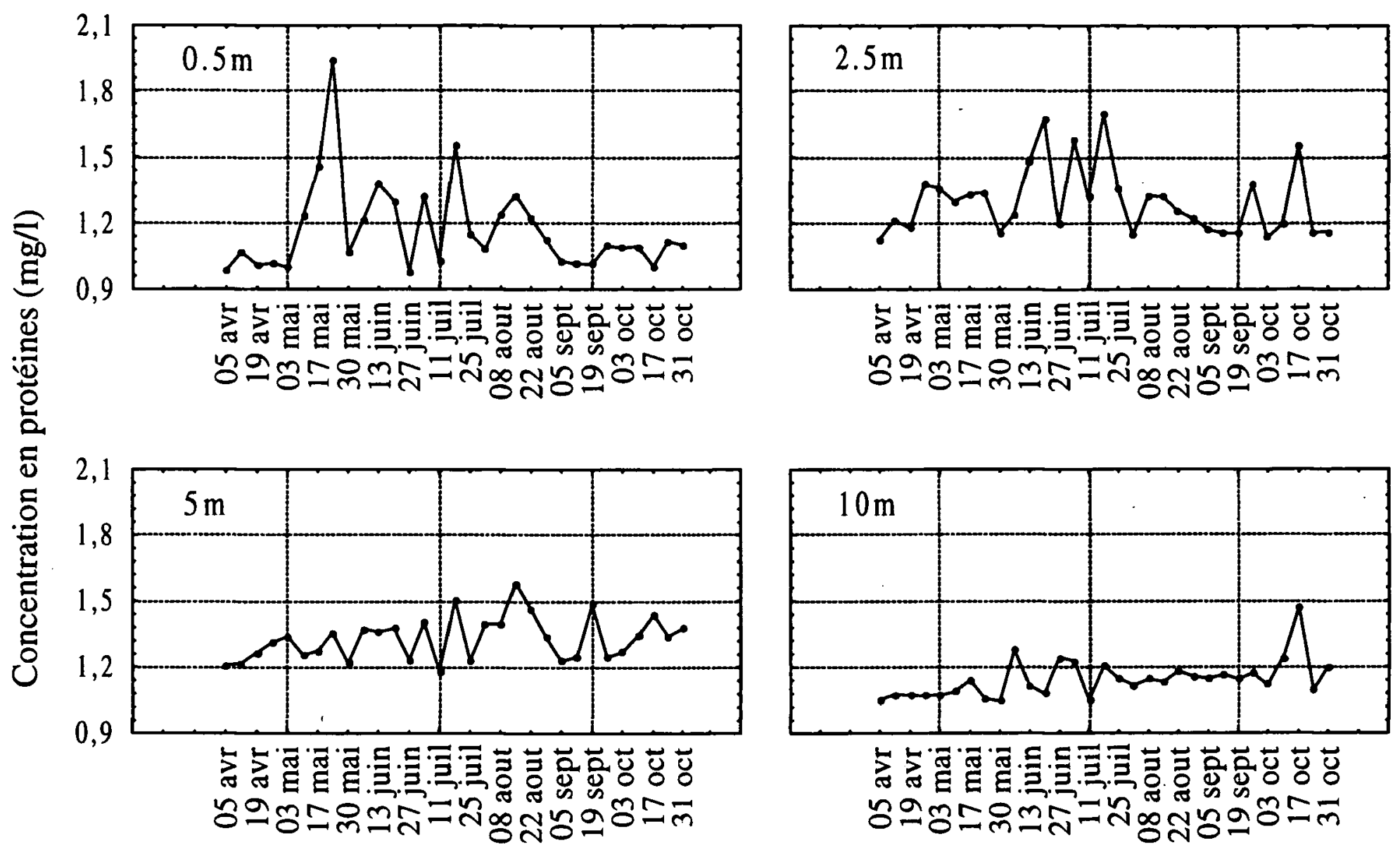

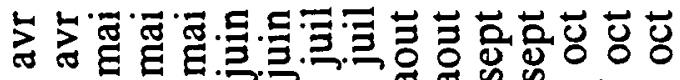

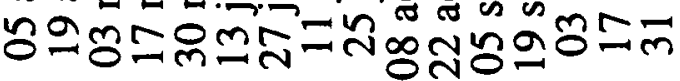

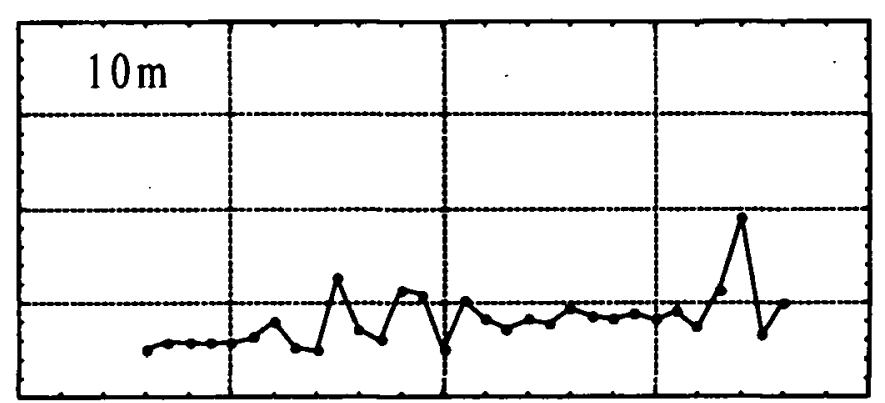

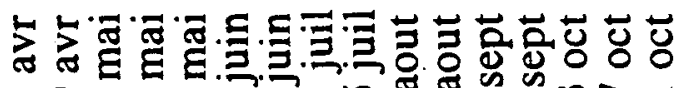

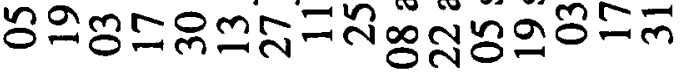

Fig. 5. Evolution spatio-temporelle des teneurs en protéines.

Fig. 5. Spatial-temporal distribution of the protein concentration.
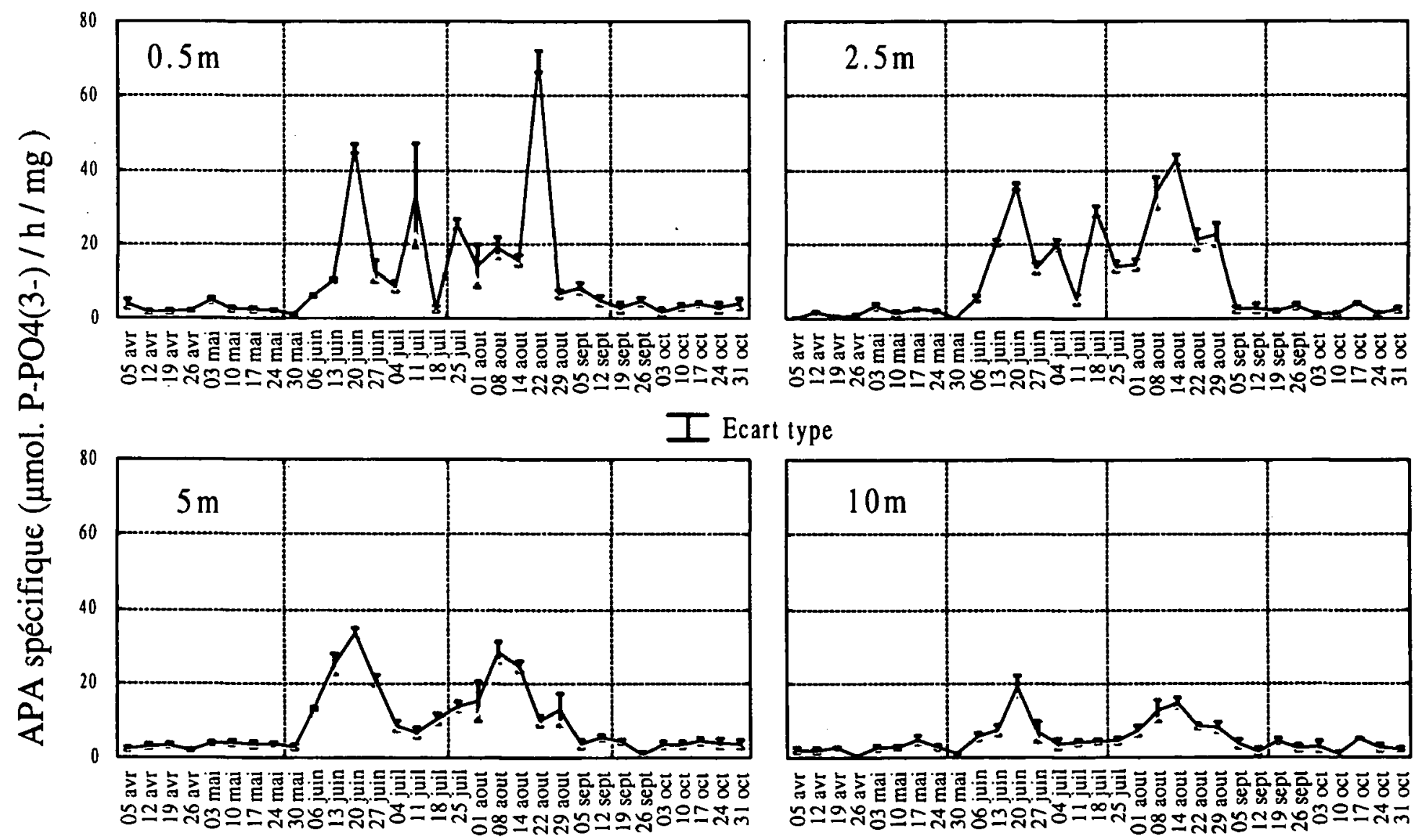

Ecart type

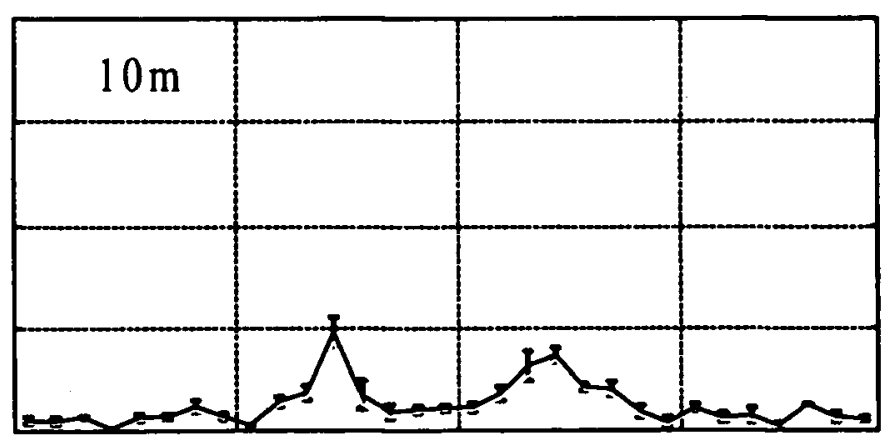

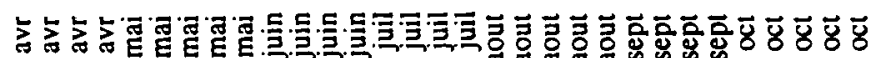

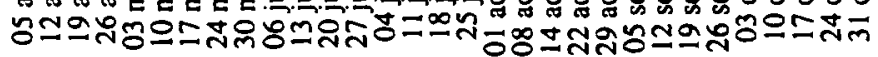

Fig. 6. Evolution spatio-temporelle de l'activité phosphatase alcaline spécifique (APA).

Fig. 6. Spatial-temporal distribution of the specific alkaline phosphatase activity (APA). 
valeurs plus élevées pendant l'été (avoisinant $40 \mu \mathrm{mol}$ $\left.\mathrm{P}-\mathrm{PO}_{4}{ }^{3-} \cdot \mathrm{h}^{-1} \cdot \mathrm{mg}^{-1}\right)$.

- D'autre part, plus on s'enfonce dans la colonne d'eau 0-10 m et plus les valeurs moyennes de l'APA spécifique sont faibles. Ainsi, cette activité enzymatique ne dépasse jamais $19,2 \mu \mathrm{mol} P-P_{4}{ }^{3-} \cdot \mathrm{h}^{-1} \cdot \mathrm{mg}^{-1}$ à - $10 \mathrm{~m}$ de profondeur alors qu'elle atteint $69,4 \mu \mathrm{mol} \mathrm{P}$ $\mathrm{PO}_{4}{ }^{3-} \cdot \mathrm{h}^{-1} \cdot \mathrm{mg}^{-1}$ en surface (Fig. 6).

\subsection{Analyse statistique}

Une matrice de corrélation a été effectuée à partir de 6 variables correspondant respectivement à la densité cyanobactérienne (Cyano), la densité des Diatomées (Diato), la densité des algues vertes (Alg. V.), la concentration en protéines (Prot), la concentration en ions orthophosphates (PO4) et l'activité phosphatasique (APA).

L'ensemble des corrélations statistiques figurent dans le tableau 1, le seuil de significativité pour $\mathrm{p}<$ 0,05 étant égal à 0,39 , après un test de Pearson. On voit tout d'abord que les espèces phytoplanctoniques prélevées l'été se trouvent en bon état physiologique puisque l'on note une corrélation significative entre les teneurs en protéines et les densités des Cyanobactéries et des algues vertes. On constate également que l'activité phosphatase alcaline est inversement corrélée et, de façon très significative, à la concentration en ions orthophosphates.

Après Analyse en Composantes Principales de ces paramètres, on constate que les deux premiers vecteurs propres traduisent $61 \%$ de la variance totale. L'opération de géométrie euclidienne consistant à projeter les variables dans un sous-espace vectoriel (Doledec \&

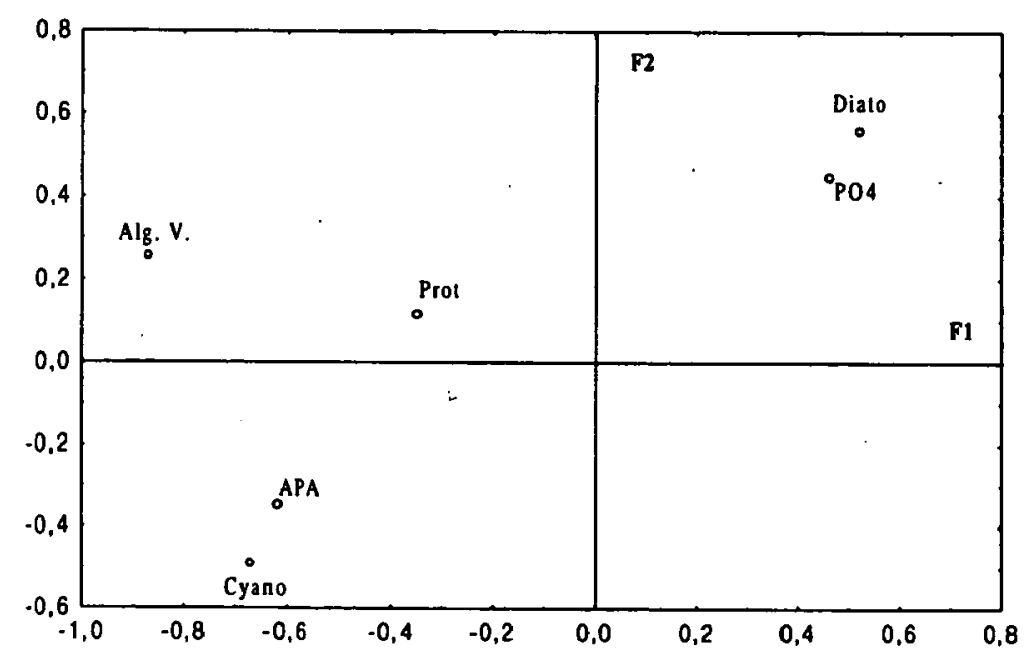

Fig. 7. Analyse en Composantes Principales : Projection des variables Cyano, Diato, Alg. V., Prot, PO4 et APA dans le sous-espace vectoriel F1-F2.

Fig. 7. Principal Component analysis : Projection of the Cyano, Diato, Alg. V., Prot, PO4 et APA variables in the F1-F2 factorial map.
Tableau 1. Corrélations entre les variables Cyano, Diato, Alg. V., Prot, PO4 et APA. Seuil de significativite : 0,39 pour $p<0,05$.

Table 1. Corrélations between Cyano, Diato, Alg. V., Prot, PO4 and APA variables. Significant : 0,39 pour $p<0,05$.

\begin{tabular}{lrrrrrr}
\hline & Cyano & Diato & Alg V. & Prot & PO4 & \multicolumn{1}{c}{ APA } \\
\hline Cyano & \multirow{2}{*}{1.00} & $-0,35$ & $\mathbf{0 , 4 0}$ & $\mathbf{0 , 6 6}$ & $-\mathbf{0 , 4 0}$ & $\mathbf{0 , 7 0}$ \\
Diato & & 1,00 & $-\mathbf{0 , 2 4}$ & 0,32 & 0,21 & $-\mathbf{0 , 3 6}$ \\
Alg. V. & & & 1,00 & $\mathbf{0 , 5 0}$ & $-0,31$ & $\mathbf{0 , 5 1}$ \\
Prot. & & & & 1,00 & $-0,38$ & $\mathbf{0 , 5 7}$ \\
PO4 & & & & & 1,00 & $-\mathbf{0 , 5 4}$ \\
APA & & & & & & 1,00 \\
\hline
\end{tabular}

Chessel 1987) permet de voir que le premier axe typiquement bipolaire oppose la densité cellulaire des algues vertes sélectionnée négativement par rapport à la densité des Diatomées et aux concentrations en ions orthophosphates sélectionnées positivement. Le deuxième axe sélectionne significativement (en valeurs négatives) la densité des cyanobactéries ainsi que l'activité phosphatase alcaline, à un degré moindre (Fig. 7).

La projection de ces variables, en fonction de la date, dans le sous-espace F1F2 montre qu'il s'agit de 2 axes saisonniers. Les descripteurs déterminants du premier axe sont, côté positif, les poussées algales printanières (donc à Diatomées) et les concentrations en $\mathrm{PO}_{4}{ }^{3-}$ élevées durant cette saison sans doute à cause des apports du fleuve. Le deuxième axe est déterminé (côté négatif) par les poussées algales estivales (algues vertes et Cyanobactéries) et l'APA (Fig. 8) .

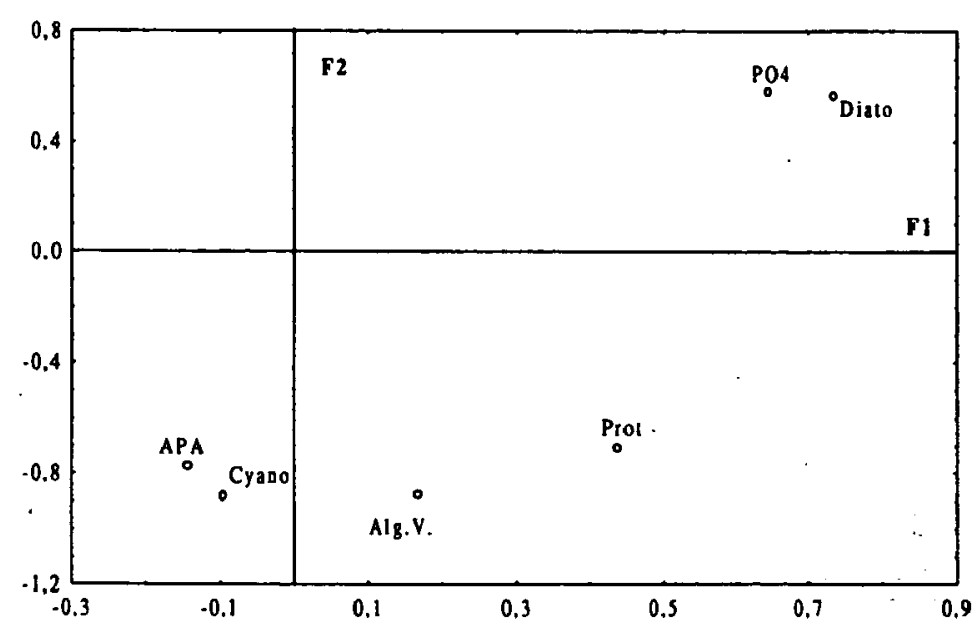

Fig.8. Analyse en Composantes Principales : Carte factorielle F1-F2 inter dates des variables Cyano, Diato, Alg. V., Prot, PO4 et APA.

Fig. 8. Principal Component analysis : F1-F2 factorial map of the Cyano, Diato, Alg. V., Prot, PO4 and APA variables depending on the dates. 


\section{Discussion et conclusion}

L'APA phytoplanctonique de la retenue de Grangent est maximale en été, minimale en automne et au printemps. Elle est principalement rattachée au phytoplancton se développant dans les premiers mètres d'eau et semble étroitement liée aux fleurs d'eau cyanobactériennes.

Ces résultats sont en accord avec ceux de Heath \& Cooke (1975) qui ont mesuré l'activité phosphatase alcaline phytoplanctonique du lac eutrophe américain East Twin. Ils ont enregistré des valeurs maximales en août et minimales en automne. Les pics estivaux coïncident avec les blooms cyanobactériens à Aphanizomenon flos aquae et les valeurs de l'APA chutent quand on descend en dessous de $4 \mathrm{~m}$ de profondeur. De nombreux autres auteurs ont démontré une nette diminution de l'APA en automne et en hiver dans des écosystèmes assez comparables à la retenue de Grangent (Healey 1973, Stevens \& Parr 1977, Taft et al. 1977, Siuda et al. 1982, Chrost et al. 1986, Currie et al. 1986, Vadstein et al. 1988, Cotner \& Wetzel 1992) mais certains ont observé, contrairement à nous, une élévation printanière de l'activité phosphatasique (Pettersson 1980, Planche-Jamet 1995).

Par ailleurs, nous avons noté une corrélation inverse entre l'APA et la teneur en orthophosphate du milieu. En effet, jusqu'à $-2,5 \mathrm{~m}$, les valeurs maximales de l'activité phosphatase alcaline sont enregistrées quand les concentrations en ions orthophosphates sont faibles $\left(<0,05 \mathrm{mg} . \mathrm{l}^{-1}\right)$. Ceci confirmerait que l'activité phosphatasique est bien indicatrice d'une carence en phosphore minéral comme l'ont montré de nombreux auteurs dont Berman (1970), Heath \& Cooke (1975), Wynne (1981), Chrost \& Overbeck (1987), Feuillade et al. (1990).

Enfin, une concordance parfaite est observée entre les pics de l'APA des couches superficielles et les maxima de densité cellulaire des Cyanobactéries. Il semble donc que les Cyanobactéries jouent un rôle prépondérant dans l'APA mesurée. L'agglomération des descripteurs APA et densité cyanobactérienne dans le sous-espace F1-F2 confirme l'idée que l'APA est étroitement liée aux fleurs d'eau cyanobactériennes (Fig. 8).

En présence d'une même concentration en orthophosphate, les algues vertes semblent plus efficaces dans l'assimilation du phosphore minéral restant que les Cyanobactéries. Ce phénomène pourrait expliquer le fait que les Cyanobactéries aient davantage recours à l'activité phosphatasique que les algues vertes ou les Diatomées. Dans un travail antérieur réalisé au labora- toire (Giraudet et al. 1997), nous avons montré une inégalité entre les espèces phytoplanctoniques dans leur comportement enzymatique. Il a été démontré, dans des conditions expérimentales identiques, que l'APA de la Cyanobactérie Microcystis aeruginosa était en moyenne plus de trente fois supérieure à celle de la Diatomée Synedra capitata. D'une façon générale, on peut penser que les algues sont de meilleures compétitrices que les Cyanobactéries dans l'assimilation du phosphore minéral disponible dans le milieu. Cette compétition pour ce type de ressource a déjà été démontrée par. Tilman (1982). Les Cyanobactéries sont donc plus souvent amenées à déclencher leur activité phosphatasique pour disposer du phosphore minéral indispensable à leur métabolisme.

Cette différence de comportement dans l'assimilation du phosphore entre les Cyanobactéries et les algues pourrait représenter un critère explicatif supplémentaire des successions écologiques.

\section{Travaux cités}

Berman T. 1970. Alkaline phosphatases and phosphorus availability in Lake Kinneret. Limnol. Oceanogr., 15 : 663-674.

Berthon J-L., Devaux J., Aleya L., Giraudet H. \& Restituito F. 1996. Déterminisme de l'eutrophisation de la retenue de Grangent (Loire): Etude des apports en nutriments, de la dynamique des populations phytoplanctoniques et des relations phytozooplanctoniques en 1990-1991. Hydroécol. Appl., 8 : 99-125.

Boavida M. J. \& Heath R. T. 1986. Phosphatase activity of Chlamydomonas acidophila Negoro (Volvocales, Chlorophyceae). Phycologia, 25 : 400-404.

Chrost R. J. \& Overbeck J. 1987. -Kinetics of alkaline phosphatase activity and phosphorus availability for phytoplankton and bacterioplankton in Lake Plussee ( North German Eutrophic Lake). Microb. Ecol., $13: 229-248$.

Chrost R. J., Wcislo R. \& Halemejko G. Z. 1986. -Enzymatic decomposition of organic matter by bacteria in an eutrophic lake. Arch. Hydrobiol., 107 : 145-165.

Cotner J. B. \& Wetzel R. G. 1992. -Uptake of dissolved inorganic and organic phosphorus compounds by phytoplankton and bacterioplankton. Limnol. Oceanogr., 37 : 232-243.

Currie D. J., Bentzen E. \& Kalff J. 1986. -Does algal-bacterial phosphorus partitioning vary among lakes? A comparative study of orthophosphate uptake and alkaline phosphatase activity in freshwater. Can. J. Fish. Aquat. Sci., 43 : 311-318.

Devaux J. 1993. Etude de la retenue de Grangent. Rapport final du Conseil Général de la Loire: 131p.

Doledec S. \& Chessel D. 1987. Rythmes saisonniers et composantes stationnelles en milieu aquatique. Acta Oecologica Oecol. Gener., $8: 403-426$.

Feder J. 1973. The Phosphatases, in Environmental phosphorus handbook. Griffith E. J., Beeton A., Spencer J. M. \& Mitchell D. T. (Ed.). Willey J. \& Sons, New York : 475-508.

Feuillade J., Feuillade M. \& Blanc P. 1990. Alkaline phosphatase activity fluctuations and associated factors in a eutrophic lake dominated by Oscillatoria rubescens. Hydrobiologia, 207 : 233-240.

Giraudet H., Berthon J-L. \& Buisson B. 1997. Comparaison de l'activité phosphatase alcaline quotidienne d'une Cyanobactérie (Microcystis aeruginosa) et d'une Diatomée (Synedra capitata). C. $R$. Acad. Sci. Paris Sciences de la vie, $320: 451-458$. 
Healey F. P. 1973. Characteristics of phosphorus deficiency in Anabaena. J. Phycol., 9 : 383-394.

Heath R. T. \& Cooke G. D. 1975. -The significance of alkaline phosphatase in a eutrophic lake. Verh. Internat. Verein. Limnol., 19 : 959-965.

Hernandez I., Niell F. X. \& Fernandez J. A. 1992. -Alkaline phosphatase activity in Porphyra umbilicalis (L.) Kützing. J. Exp. Mar. Biol. Ecol., 159 : 1-13.

Hino S. 1988. Fluctuation of algal alkaline phosphatase activity and the possible mechanisms of hydrolysis of dissolved organic phosphorus in lake Barato. Hydrobiologia, 157 : 77-84.

Hotelling H. 1933. Analysis of a complex of statistical variables into principal components. J. Educ. Psychol., 29 : 417-520.

Huber A. L. \& Hamel K. S. 1985. Phosphatase activities in relation to phosphorus nutrition in Nodularia spumigena (Cyanobacteriaceae). 1 Field studies. Hydrobiologia, $123: 145-152$.

Jamet D., Aleya L. \& Devaux J. 1995. Diel changes in the alkaline phosphatase activity of bacteria and phytoplankton in the hypereutrophic Villerest reservoir (Roanne, France). Hydrobiologia, 300-301: 49-56.

Lapointe B. E. \& O'Connel J. 1989. Nutrient-enhanced growth of Cladophora prolifera in Harrington Sound, Bermuda : Eutrophication of a confined, phosphorus limited marine ecosystem. Est. Coast. Shelf. Sci., 28 : 347-360.

Legendre L. \& Watt W. D. 1972. On rapid technique for plankton enumeration. Ann. Inst. Oceanogr. Paris, 58 : 173-177.

Legendre L. \& Legendre P. 1979. -Le traitement multiple des données écologiques, In Ecologie numérique, tome 1. Masson P. U. Q. : $197 p$.

Lohman H. 1908. Untersuchungen zur Feststellung des Vollständigen Gehaltes des Meeres an Plankton. Wiss. Meeresunters. Abt. Kiel N. F., $10: 132-170$.

Lorenzen C. J. 1967. Determination of chlorophyll and pheopigments : spectrophotometric equations. Limnol. Oceanogr., 12 : 343-346.

Lowry O. H., Rosebrough N. J., Farr A. L. \& Randall R. J. 1951. Protein Measurement with the Folin Phenol Reagent. J. Biol. Chem., 193 : 265-275.

Michard M. 1994. Etude du déterminisme des blooms à Microcystis aeruginosa au sein de la retenue hypereutrophe de Villerest (Roanne, France): Dynamique des communautés phyto-zooplanctoniques, composition biochimique du matériel particulaire, contenu énergétique des zooplanctontes. Thèse de 3ème Cycle, Univ. Blaise Pascal, Clermont-Ferrand : 269 p.
Olsson H. 1991. Phosphatase activity in an acid, limed Swedish la$\mathrm{ke}$, In Microbial enzymes in aquatic environments. Chrost R. J. (Sc. Ed.). Springer Verlag, New York : 206-219.

Perez M. \& Romero J. 1993. Preliminary data on alkaline phosphatase activity associated with mediterranean seagrasses. Bot. Mar., $36: 499-502$.

Pettersson K. 1980. Alkaline phosphatase activity and algal surplus phosphorus as phosphorus-deficiency indicators in lake Erken. Arch. Hydrobiol., 89 : 54-87.

Planche-Jamet D. 1995. Variations temporelles et verticales de l'APA associée à différentes classes de taille du matériel particulaire au sein de 3 lacs du Massif Central de degrés trophiques différents(lac Pavin, lac d'Aydat, retenue de Villerest). Thèse de Doctorat en Biologie, Univ. Blaise Pascal, Clermont Ferrand : 179 p.

Reichardt W., Overbeck J. \& Steubing L. 1967. -Free dissolved enzymes in lake waters. Nature, $216: 1345-1347$.

Siuda W. \& Chrost R. J. 1987. The relationship between alkaline phosphatase (APA) activity and phosphate availability for phytoplankton and bacteria in eutrophic lakes. Acta Microbiol. Pol., $36: 247$ - 257 .

Siuda W., Chrost R. J., Wcisto R. \& Krupka M. 1982. Factors affecting alkaline phosphatase activity in a lake (short-terms experiments). Acta Hydrobiologia, 24 : 3-20.

Stevens R. J. \& Parr M. P. 1977. The significance of alkaline phosphatase activity in Lough Neagh. Freshwat. Biol., $7: 351$-355.

Taft J. L., Loftus M. E. \& Taylor W. R. 1977. Phosphate uptake from phosphomonoesters by phytoplankton in the Chesapeake Bay. Limnol. Oceanogr., 22 : 1012-1021.

Tilman D. 1982. -Ressource competition and community structure, In Population biology 17. Princeton University Press, Princeton: $296 \mathrm{p}$.

Utermöhl H. 1958. -Zur Vervolkommung der quantitative Phytoplankton. Methodik. Mitt. Intern. Ver. Limnol., 9 : 1-38.Vadstein O., Jensen A., Olsen Y. \& Reinersten H. 1988. -Growth and phosphorus status of limnetic phytoplankton and bacteria. Limnol. Oceanogr., $33: 489-503$.

Wynne D. 1981. -Phosphorus, phosphatases and the Peridinium bloom in Lake Kinneret. Verh. Internat. Verein. Limnol., 21 : 523 527.

Wynne D. \& Rhee G. Y. 1986. -Effects of light intensity and quality on the relative $\mathrm{N}$ and $\mathrm{P}$ requirement (the optimum $\mathrm{N}$ : $\mathrm{P}$ ratio) by marine planktonic algae. J. Plankton. Res., 8 : 91-103. 